\title{
New energy-saving technologies in transport energy
}

\author{
E. Lazarev \& G. Lomakin \\ Department of Internal Combustion Engines, \\ South Ural State University, Chelyabinsk, Russia
}

\begin{abstract}
Diesel engines being self-contained power plants are the most promising energy sources in transport from the point of view of saving natural resources. In order to improve the fuel efficiency and power density of diesel engines for further development of transport energy, requires using energy-saving technologies.

Such technologies to create diesel engines are based on a comprehensive improvement and optimization of operating cycle and its comprising processes. Special attention is paid to ensuring the effective combustion of fuel within the constraints imposed by the emission of harmful substances in the exhausting gases and the thermo-mechanical loaded parts.

In order to evidence-based selection, method of management of combustion process on the basis of analysis of physical and chemical characteristics of processes developed intracylinder refined mathematical model of combustion of fuel in diesel engines.

The basis of determining efficiency of selection and management of combustion process is a differentiated approach to the assessment of the control actions. According to the results of computational and experimental synthesis cycle in diesel engines, basic principles of fuel control combustion process were developed.

Implementation of the developed guidelines and best management practices during the combustion process of fuel improves technical level and competitiveness of diesel engines, improves fuel efficiency by $5 \ldots 7 \%$, reduces thermo-mechanical loading, maximum temperature and pressure of gas in
\end{abstract}


cylinder by $5 \ldots 6 \%$ and emissions including - particulate matter (soot) $-25 \ldots$ $30 \%$ and nitrogen oxides $-18 \ldots 20 \%$, common and high levels noise of operating diesel reduces by $2 \ldots 2.5 \%$ and by $5 \ldots 7 \%$, respectively.

Keywords: diesel, fuel economy, duty cycle, methods and principles of management of the process combustion of fuel.

\section{Introduction}

Energy-saving technologies increasing power density, fuel efficiency, service life and reducing emissions of harmful substances with exhaust gases are required to improve diesel competitiveness in transport and traction machines. Highpowered diesels in traction machines (class of 6-50 tonnes of fraction) should guarantee modern techno-economic and environmental indicators: specific fuel consumption not exceeding $205 \mathrm{~g} / \mathrm{kWh}$; oil consumption through burning -0.02 ... $0.05 \%$ of fuel consumption; torque margin not less than $30 \%$; service life not less than 18000 moto-hours; emissions at Tier-4 and UNECE Regulations No. 96 and No. 97/68 $(6.0 * 3.5 * 1)$; unification of main construction elements [1].

Energy-saving technologies in diesel engines are aimed at improving processes that make up an operating cycle. A special attention is paid to optimization of fuel combustion parameters applying scientifically grounded methods of combustion process control.

\section{Thermal and mechanical loading of diesel}

Thermal loading includes thermal effects (heat flows, attacks of hot gases and the cooling environment) on the details of a working medium in the cylinder and temperature levels achieved during engine operation. The most loaded regarding the temperature are pistons, cylinder head, fuel injector nozzles and exhaust valves. The large temperature difference is the cause of high temperature stress.

Mechanical loading, characterized by the pressure of gases, fuels, lubricants and cooling liquids, inertial forces, exacerbates and complicates the condition of thermally loaded components. High pressure forces from the load imposed on the gas temperature impact that the presence of stress concentration zones resulting in cracking. Thermal and mechanical loads increase with increasing power density and an increase in the diameter of the cylinder. Excess temperature and mechanical loadings lead to a significant increase of detail wears and reduction of service life of a diesel engine.

\section{Emissions of harmful substances in exhaust gases}

The main normalized components of harmful substances in exhaust gases are particulate matter (soot), carbon monoxide, nitrogen oxide and unsaturated hydrocarbons. The formation of these components occurs in the combustion 
process and is closely associated with features of fuel supply, mixing formation, ignition and fuel burn-out.

The increase of combustion process efficiency and as a consequence fuel economy, involves an increase of pressure and temperature of working substance in the diesel's cylinder, reduction of emissions of particulate matter (soot) and carbon monoxide. However, these conditions are favorable for the formation of nitrogen oxides. Therefore, increasing fuel efficiency and reducing emissions of nitrogen oxides in diesel engines are conflicting factors, that's why their optimal combination is achieved through compromise.

\section{Improvement of fuel combustion process}

For improvement of diesel working cycle it's reasonable to increase controllability of a combustion process based on the choice of control methods. The system of improvement of fuel combustion process is based on a unified methodological concept, involving a differentiated approach to the assessment of control actions on specific periods and the use of released heat. Scientifically grounded choice of methods for combustion process control is based on a multicriteria evaluation of controllability depending on the purpose of working cycle improvement.

\subsection{Physical and chemical characteristics fuel combustion process}

Fuel combustion is defined by processes of its distribution in a combustion chamber, heating, evaporation, mixing with air and oxidation process, which is a chemical chain reaction. The intensity and uniformity of fuel-air mixture is defined by diffusion processes and heat exchange. After the injection due to the intense heat extraction in the walls of intracylinder space the appearance of flame - "apparent" combustion - is delayed. In the process of fuel combustion there are initial and main periods which differ from each other in mechanisms of flame spreading.

The speed in the initial combustion period depends on the amount of fuel vapor, rate of pre-flame reactions, quantity, scale, distribution of self-ignition centers and turbulent flame in the mixture. The combustion in the flame front depends on fuel properties, the combustion chamber, charge air traffic intensity, etc.

Vapors coming from local zones after the process of fuel injection are burnt in the main period. The main period combines advanced combustion and afterburning. The burn-up rate depends on the intensity of the diffusion of the mixture components in the area of chemical reactions and the local air excess ratio in the flame zone and the reaction rate is determined by fuel properties and the diesel operation.

\subsection{Control and controllability of fuel combustion processes}

The effectiveness of a working cycle depends on the applied methods of combustion process control, which represent a set of effects on distribution, 
vaporization, ignition, nature and duration of fuel burn-out in the mixture, as well as the use of released heat.

Control elements are singled out in the combustion process as a system of control: state of the air-fuel charge, composition and condition of the fuel-air mixture, methods and conditions of its formation, ignition and combustion. The properties of control elements are characterized by control parameters: pressure, temperature and intensity of a vortex motion of air charge, excess air ratio, pressure, nature and duration of fuel injection, ratios structural elements of the combustion chamber, number and diameter of the spray nozzle holes, etc. The rest of the system is a control object, namely, timeliness, specificity, monotony, and duration and adiabaticity of fuel combustion process.

Global goal of control is an achievement of optimal values of main parameters of the combustion process in aggregate: timeliness, specificity, monotony and duration, minimization of heat loss - adiabaticity. During complicated burn-out mechanism there could be local control objectives achievement of optimal values of an individual parameter in the combustion process.

The criterion of control is used to compare control actions. Controllability of the combustion process is the ability of law fuel burn-out in the mixture and use of released heat to react to a change of control actions. The criterion of controllability in the combustion process determines the extent of correspondence of the real and required laws of fuel burn-out in the mixture and the use of released heat at a specific control action. The optimal control problem is to find and implement a type of control action in which the criterion of control takes the most advantageous value.

Improving the fuel combustion process should be considered as differential effects on a set of parameters, consistently reflecting the changes of air-fuel mixture and its elements. A differentiated approach as a basis to improve the combustion process suggests its systematization, classification and evaluation of the effectiveness of control methods. The complex of parameters for combustion process is determined by the nature and mechanisms of fuel burn-out and is reflected in mathematical modeling.

\subsection{Mathematical modeling of fuel burn-out}

The author's mathematical model of the combustion process [2], a main element of which is the description of characteristics of a complex type fuel burn-up $x=f(\varphi)$, enables us to evaluate the effectiveness of a differentiated approach to the control on the basis of numerical experiments

$$
x=1-\exp \left[\tilde{n}\left(\frac{\varphi}{\varphi_{i}}\right)^{m_{i}-m_{\hat{i}}}\left(\frac{\varphi}{\varphi_{z}}\right)^{m_{i}+1}\right] \text {, if } \varphi \succ \varphi_{i}, m_{i}=m_{\hat{i}},
$$

where $x$ - a share of fuel burnt to the crankshaft angle $\varphi$; $\varphi_{H}, \varphi_{z}-$ a duration of the initial period and the process of combustion, degree rotation of the crankshaft; 
$m_{\mathrm{H}}, m_{\mathrm{o}}$ - parameters of a combustion character in the initial and main periods of the combustion process.

Besides, dependence of the duration $\left(\varphi_{z}\right)$ combustion process on the character of the combustion periods and combustion efficiency $(\xi)$ ratio on the length of the combustion process, the composition of the mixture $(\alpha)$ and speed of the crankshaft diesel are included in the mathematical model [2].

\subsection{Criterion evaluation of the effectiveness of control methods}

Evaluation criterion provides scientifically grounded selection of the optimal method of control of the combustion process in improving the working cycle diesel. Performance criteria for the formation of the methods are used by the parameters of the mathematical model of combustion process. Dimensionless parameters are used as criteria. These parameters characterize the correspondence of actual parameter (or set of parameters) of a combustion process to the optimal value. Particular criteria of controllability are used

$$
k_{i}=\frac{\left|i_{\text {opt }}-i\right|}{i_{\text {opt }}},
$$

where $i, i_{\text {opt }}$ - actual and optimal parameters combustion process, followed by a complex criterion that estimate performance characteristics (power, fuel economy, mechanical and thermal loading of the elements emissions exhaust gas) of a diesel engine

$$
K_{i}=\sum_{i=1}^{n} w_{i} k_{i},
$$

where $w_{\mathrm{i}}-i$ priority coefficient; $k_{\mathrm{i}}-i$ partial criterion; $n-$ the number of particular criteria.

Priority coefficients are determined by numerical and theoretical, experimental method or expert estimates.

On the basis of quantitative and qualitative assessments of controllability of control methods combined by a single character of control actions into classification groups there is a hierarchical structure on the level of control and the degree of controllability to narrow the search [2].

The level of control of the combustion process is determined by the degree of change in the criteria for the group of controllability methods. There are three levels of management: high, medium and low. In the transition from a higher level to a subsequent one the degree of controllability criteria change is reduced.

Comparative estimation of absolute values of the criteria is used to determine the degree of controllability of a combustion process in a group of control methods. There are three degrees of control: high, medium and low.

\subsection{Principles of control of the combustion process in diesel engines}

Numerical modeling of the working cycle was performed using a mathematical model of the combustion process, which is based on the equation (1). In the end, 
basic principles of combustion process control, reflecting characteristics of the intensity of impact of fuel burn-out on diesel engine performance indicators are developed:

- The differential change in the intensity of fuel burn-out in the initial and main periods of the combustion process provides the managing impacts on the pressure and temperature of the working fluid in the cylinder, power, fuel economy and emissions of hazardous substances with exhaust gases;

- Changes in the intensity of fuel burn-out during the initial period of the combustion process with the restriction period, it mainly provides a control action on the pressure, temperature of the cylinder working body and the emissions of hazardous substances with exhaust gases of the same power and fuel economy of the diesel engine;

- Changes in the intensity of fuel burn-out in the fundamental period of the combustion process with the restriction in its initial period has manipulated by power and fuel economy of the diesel engine with a predominant influence on the temperature of the working body in the cylinder;

- Redistribution of the intensity of fuel burn-out between the initial and main periods of the combustion process has a control action on monotony of the combustion process based on pressure and temperature of the working body in the cylinder, power and fuel economy of the diesel engine;

- Improvement of the combustion process is achieved by optimizing monotonous length and nature of fuel combustion process providing compromise limits of pressure and temperature of the working body in the cylinder, power, fuel economy of and emissions of hazardous substances with exhaust gases.

High level control is characterized by complex criteria dependent on the composition of the mixture, medium - on the mixture condition, method and conditions of its formation, as well as ignition conditions.

Modern control methods do not guarantee a high degree of controllability that indicates that the reserves of combustion process improvement. Reducing thermal losses (providing adiabatic) of the combustion process is achieved by influence on the mixture composition. Reducing the duration of exposure to combustion is provided on the condition of air-fuel mixture, and the timeliness and specificity of fuel burn-out - the effects on the conditions ignition of the mixture.

Changing the status of air-fuel mixture control is achieved by improving the intensity of the mixing process and the distribution of a mixture of the combustion chamber. Improving conditions in the ignition of fuel mixture is achieved by changing the law of fuel supply - driven, in particular the split, its injection.

Implementation of the basic principles of control methods and the achievement of high level essentially of controllability combustion process will improve fuel efficiency by $5 \ldots 7 \%$, reduce the maximum pressure and temperature of the gas in the cylinder by $5 \ldots 6 \%$, emissions of soot by $-25 \ldots$ $30 \%$ and oxides nitrogen by $-18 \ldots 20 \%$, and the total level of high frequency noise by $2 \ldots 2.5$ and $5 \% \ldots 7 \%$. 


\section{Conclusion}

Improving the working cycle in regard to the increase of power density and fuel efficiency is accompanied by an increase of thermal and mechanical loading of parts (lower resource) and emissions of diesel exhaust gases. The use of new energy-saving technologies guarantees a compromise solution for raising the technical level of diesel engines.

\section{References}

[1] Murzin, V.S. Improving working process T-Series diesel engines to achieve the parameters of the global technological level. Engine building, 2 (244), pp. 17-21, 2011.

[2] Lazarev, E.A. Modeling of the combustion process in diesel engines (Chapter 2). Topics in Basic principles, methods and efficiency of means for improving the efficiency of fuel combustion process to increase the technical level of tractor diesel engines, ed. E.A. Lazarev, Publishing Center SUSU: Chelyabinsk, pp. 27-49, 2010. 